\title{
A História dos modelos de Eugenio Beltrami para a geometria hiperbólica
}

\author{
Márcio Rostirolla Adames (D) Fernanda Mocelin Schena (D)
}

\section{Resumo}

Eugenio Beltrami foi o primeiro a apresentar superfícies nas quais valem os resultados contraintuitivos da geometria não euclidiana, todavia seu nome frequentemente é preterido na história do surgimento dessa geometria. O presente trabalho objetiva contar a história dos modelos apresentados por Beltrami, que concretizaram a geometria axiomática desenvolvida por Bolyai e Lobachevsky. Esses modelos viriam a ser conhecidos por modelo de Klein e os modelos (do disco e do semiplano) conformais de Poincaré. O trabalho ainda descreve, brevemente, as contribuições de Klein e Poincaré, como também apresenta a realização moderna da geometria hiperbólica no hiperboloide.

Palavras-chave: História da Matemática; Geometria Hiperbólica; Eugenio Beltrami.

\begin{abstract}
Eugenio Beltrami was the first to present surfaces in which the counterintuitive results of nonEuclidean geometry hold, however, his name is often overlooked in the history of the emergence of this geometry. The present work aims to tell the story of the models presented by Beltrami, which materialize the axiomatic geometry developed by Bolyai and Lobachevsky. These models would come to be known as the Klein model and the Poincaré (disc and half-plane) conformal models. Moreover, the paper briefly describes the contributions of Klein and Poincaré as well as presents the modern realization of hyperbolic geometry in the hyperboloid.
\end{abstract}

Keywords: History of mathematics; Hyperbolic Geometry; Eugenio Beltrami.

\section{Introdução}

Euclides reuniu, em uma estrutura lógica unificada, muitos dos resultados da geometria conhecidos em sua época através do método axiomático, no qual a validade de cada afirmação é verificada por uma sequência lógica de deduções a partir de axiomas e postulados, que deveriam ser aceitos previamente ${ }^{1}$ pelo leitor, sem levantar muita controvérsia. Os axiomas e postulados deveriam ser verdades simples e autoevidentes. O quinto desses postulados, denominado postulado das paralelas, é famoso por não ser tão simples quanto se desejaria para um axioma ou postulado, como podemos verificar na tradução ${ }^{2}$ proposta por Bicudo [7]:

\footnotetext{
${ }^{1}$ Diferentemente da visão moderna dos axiomas, como premissas a partir das quais derivam-se consequências.

${ }^{2} \mathrm{O}$ termo reta era utilizado para o que chamamos hoje de segmento de reta. Euclides não tinha um conceito de reta infinita definido.
} 
$5^{\mathbf{o}}$ postulado - Também, caso uma reta, encontrando duas retas, faça ângulos interiores e sobre os mesmos lados, menores do que dois retos; sendo prolongadas ilimitadamente, as duas retas vão se encontrar sobre o lado em que estão os menores do que dois retos.

Desde a antiguidade, muitos autores buscaram equivocadamente demonstrar esse postulado em termos dos demais ou apresentar uma formulação equivalente. Contudo, as pretensas demonstrações sempre apresentavam erros ou subentendiam hipóteses adicionais não enunciadas.

Uma estratégia utilizada foi assumir a validade dos demais postulados e, negando a validade do quinto, encontrar uma contradição. Na busca por tal contradição, o matemático italiano Giovanni Girolamo Saccheri (1667 — 1733) e o matemático suíço Johann Heinrich Lambert (1728 - 1777) desenvolveram, independentemente, vários resultados e concluíram que a validade do postulado das paralelas estava relacionada aos ângulos superiores de determinados quadriláteros. Esses quadriláteros viriam a ser conhecidos, respectivamente, como quadriláteros de Saccheri e de Lambert. Eles obtiveram sucesso parcial considerando os casos de os ângulos superiores serem obtusos (equivalente a não haver nenhuma paralela a uma reta por um ponto fora dela), agudos (equivalente a existirem infinitas retas paralelas a uma reta dada passando por um ponto fora dela) ou retos (equivalente à validade do quinto postulado). No caso do ângulo obtuso, conseguiram encontrar uma contradição bem fundamentada, mas falharam em fazê-lo no caso do ângulo agudo. Ao longo desse processo, desenvolveram alguns resultados que não dependiam do postulado das paralelas ou que assumiam a hipótese do ângulo agudo.

Nas primeiras décadas do século XIX, Gauss ${ }^{3}$, Bolyai e Lobachevsky, de modo independente, deduziram muitos resultados geométricos a partir da validade dos quatro primeiros postulados de Euclides e da hipótese de haver mais de uma paralela a uma reta passando por um ponto fora dela, mas sem encontrar uma contradição com a hipótese do ângulo agudo. Com isso, acabaram por desenvolver um sistema axiomático, aparentemente consistente, mas sem mostrar que tal sistema aplicava-se a qualquer coisa do mundo real. Uma exploração detalhada dos desenvolvimentos relacionados ao quinto postulado pode ser encontrada no livro [21].

No mesmo período, mas sem relação com os trabalhos referentes ao quinto postulado, ocorreram muitos desenvolvimentos na geometria diferencial, como o estudo das superfícies de curvatura constante e negativa. Isso acabou permitindo que fossem encontrados modelos da geometria de Bolyai e Lobachevsky, que até então não se relacionava com outros ramos da matemática, conforme destacado em [15]:

\begin{abstract}
Nos primeiros quarenta anos de sua história, o campo da geometria não euclidiana existia em uma espécie de limbo, divorciado do resto da matemática e sem qualquer fundamentação consistente. Entretanto, a teoria das superfícies curvas de Gauss (1827) e a teoria das variedades n-dimensionais de Riemann (1868), forneceram uma maneira de integrar a geometria não euclidiana em ramos mais respeitáveis da Matemática. (MILNOR, 1982, p. 10, tradução nossa)
\end{abstract}

Essa integração do campo da geometria não euclidiana com a Matemática ocorreu graças aos trabalhos do matemático italiano Eugenio Beltrami. Nos anos de 1868 e 1869, Beltrami publicou dois artigos apresentando superfícies cujas geometrias satisfazem o sistema de axiomas de Bolyai e

\footnotetext{
${ }^{3}$ Que não publicou os resultados, mas deixou evidências de seu interesse no assunto em cartas trocadas com diversos matemáticos.
} 
Lobachevsky e parametrizações dessas superfícies no plano, que são conhecidas atualmente como Disco de Klein, Disco de Poincaré e Semiplano de Poincaré.

Desse modo, Beltrami materializou o mundo contraintuitivo das geometrias não euclidianas na geometria de superfícies de curvatura gaussiana constante e negativa, as quais chamou de pseudoesferas. As parametrizações das superfícies de curvatura constante e negativa permitem representá-las em regiões concretas do plano, gerando modelos para a geometria não euclidiana.

Além disso, a existência de tais modelos implica, indiretamente, a independência do quinto postulado em relação aos outros axiomas, pois é possível obter um sistema aparentemente consistente de axiomas a partir da sua negação.

O propósito do presente trabalho é divulgar os trabalhos de Beltrami e contextualizá-los na história da geometria não euclidiana. Essa contextualização permite ver os modelos de geometria hiperbólica como representações da geometria de uma superfície concreta, do ponto de vista da geometria diferencial, e não apenas como exemplos de geometrias nas quais não vale o quinto postulado, desprovidas de significado per se.

O trabalho não pretende desenvolver toda a história da geometria não euclidiana em detalhe e nem explorar os objetos geométricos de interesse nos modelos dessa geometria, mas focamos nas contribuições de Beltrami. Há diversos trabalhos que abordam com maior detalhe a geometria dos modelos, como [9] e [8].

Os predecessores de Beltrami perceberam, no caso da não validade do postulado, a importância de "pontos no infinito", formando uma espécie de fronteira para a geometria. Beltrami tornou essa fronteira concreta e acessível em seus modelos. Entre outros resultados, ele mostrou que a trigonometria das geodésicas da pseudoesfera estava de acordo com todos os resultados deduzidos a partir da negação do quinto postulado.

Os modelos criados por Beltrami foram integrados a outros contextos bem estabelecidos da matemática, posteriormente, por Klein, Liouville e Poincaré, motivo pelo qual os três últimos são vinculados aos nomes dos modelos criados originalmente por Beltrami.

Por fim, o trabalho apresenta a realização moderna desses modelos, como projeções naturais do hiperboloide no espaço de Minkovski, popularizada após a introdução da relatividade geral de Einstein.

\section{Eugenio Beltrami}

O matemático italiano Eugenio Beltrami (1835 - 1900) nasceu em Cremona e era filho do homônimo Eugenio Beltrami, um iluminador e gravador de pedras, e de Elisa Barozzi, descendente de uma antiga família veneziana e mulher de sensibilidade e cultura musical relevantes.

Ele frequentou a Universidade de Pavia durante três anos, concluindo parte do curso de Engenharia Ferroviária em 1856. Teve como professores os renomados matemáticos italianos Antonio Maria Bordoni e Francesco Brioschi, que chegou a ser presidente da Academia Nacional do Lincei e senador do Reino. Beltrami foi expulso da faculdade por manter envolvimento com o movimento de Ressurgimento, uma manifestação popular que buscava unificar a Itália entre os anos de 1815 e 1870.

Após a expulsão da universidade, Beltrami não pôde manter a dedicação exclusiva aos seus estudos e começou a trabalhar na companhia ferroviária de Lombardo-Vêneto. Devido ao cuidado 
Figura 1: Retrato de Eugenio Beltrami (domínio público).

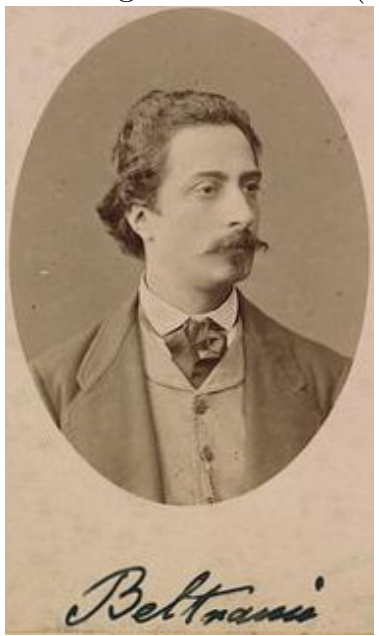

incessante e à influência de Brioschi, Beltrami conseguiu manter suas atividades acadêmicas paralelamente à vida profissional.

Sua primeira posição na universidade foi como professor na Universidade de Bolonha, em 1862. Depois, passou a Pisa e retornou a Bolonha no ano de 1866, compondo o Departamento de Mecânica Racional. Em 1873 mudou-se para Roma e depois, em 1876, para Pavia, atuando como professor nas respectivas universidades nos campos de física-matemática e mecânica superior. Permaneceu em Pávia por quinze anos, até retornar a Roma em 1891, onde faleceu em fevereiro de 1900.

É importante destacar que a física-matemática da época de Beltrami formava um campo de pesquisa muito avançado, abrangendo teorias matemáticas que levariam a descobertas da Física e suas novas vertentes, incluindo a teoria do calor, fenômenos da eletricidade e da elasticidade, entre outros. Esse artigo não pretende detalhar toda a contribuição de Beltrami à ciência, mas procura descrever os estudos relacionados à geometria não euclidiana, com ênfase nos trabalhos sobre espaços de curvatura constante e negativa.

\section{Precursores dos modelos: projeções de geodésicas.}

As grandes expedições das potências europeias dos séculos XV e XVI tornaram a cartografia tema importante no continente e muitos matemáticos contribuiram para seu desenvolvimento. Embora não faça parte do escopo deste trabalho detalhar tal área, ressaltamos uma observação de Lagrange sobre projeções da esfera no plano, no artigo "Sur la construction des cartes géographiques" [14]:

Se o centro [da projeção] está no centro da esfera, a projeção é denominada central e ela tem a propriedade que todos os grandes círculos encontram representados por linhas retas (...) (LAGRANGE, 1779, p. 638, tradução nossa)

A observação de Lagrange pode ser verificada ao recordarmos que os grandes círculos são obtidos pela interseção da esfera com planos que passam por seu centro, de modo que se projetarmos a superfície da esfera a partir do seu centro, sobre um plano qualquer, encontraremos a projeção de 
Figura 2: Projeção de uma geodésica da esfera em uma linha reta no plano.

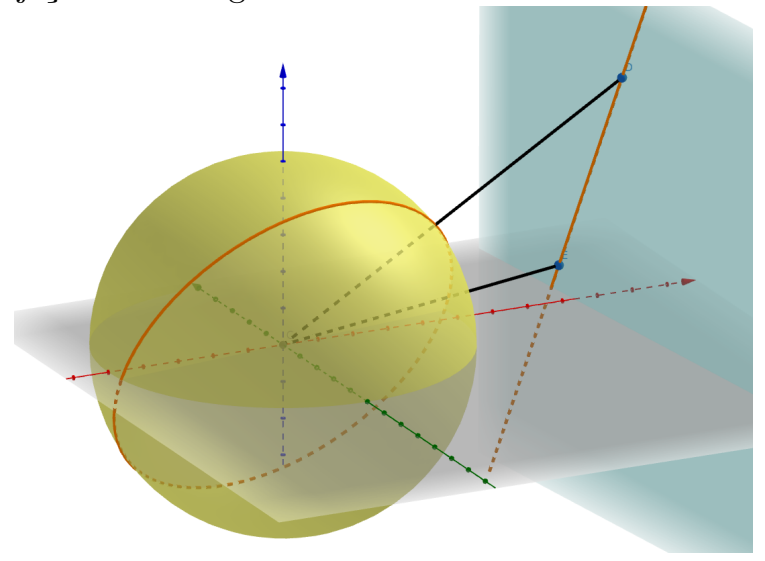

uma geodésica na interseção do plano que a define com o plano de projeção, obtendo sempre uma linha reta.

Em "Risoluzione del problema: Riportare $i$ punti di una superficie sopra un piano in modo che le linee geodetiche vengano rappresentate da linee rette" ([4]), Beltrami cita o artigo de Lagrange e investiga quais superfícies podem ser parametrizadas de modo que suas geodésicas sejam exatamente as retas no plano e chega a duas conclusões:

1. a superfície deve ter curvatura constante e positiva;

2. no caso de uma curvatura positiva, a métrica de tal superfície é dada, nas coordenadas (u,v), por

$$
\mathrm{ds}^{2}=\mathrm{R}^{2} \frac{\left(\mathrm{a}^{2}+\mathrm{v}^{2}\right) \mathrm{du} \mathrm{u}^{2}-2 u v d u d v+\left(\mathrm{a}^{2}+\mathrm{u}^{2}\right) d v^{2}}{\left(\mathrm{a}^{2}+\mathrm{u}^{2}+\mathrm{v}^{2}\right)^{2}},
$$

onde a e $\mathrm{R}$ são constantes reais.

Pode-se verificar diretamente que essa é a métrica obtida pela projeção de uma esfera de raio R, a partir do seu centro, em um plano que está a uma distância a do centro da esfera.

Não há indicações de que Beltrami buscasse nesse trabalho um modelo para a geometria não euclidiana de Bolyai e Lobachevsky. Contudo, cabe ressaltar que isso não seria possível: a geometria dessa projeção é a geometria da esfera, na qual vale a hipótese do ângulo obtuso para os quadriláteros de Lambert e Saccheri, e, portanto, apresenta contradições com os outros postulados.

\section{Os modelos de Beltrami}

Na Itália, durante a década de 1860, o assunto das geometrias não euclidianas adquiria certa visibilidade. Em 1863, o professor de geometria superior na Universidade de Nápoli, Giuseppe Battaglini (1826 - 1894), fundou o "Giornale di matematiche", no qual foram publicados diversos artigos sobre a geometria não euclidiana, incluindo o primeiro trabalho de Beltrami intitulado "Saggio di interpretazione della geometria non-euclidea" [5], o qual chamaremos resumidamente de Saggio. Beltrami introduziu o primeiro modelo de geometria não euclidiana nesse artigo. 
Entre 1868 e 1869, em dois artigos influentes, Beltrami forneceu modelos da geometria não euclidiana de Lobachevsky e Bolyai. Um desses modelos é mais conhecido por modelo de Klein, outro como modelo do disco de Poincaré, o terceiro como o modelo do semiplano de Poincaré. Um quarto, que Beltrami desenvolveu, como o modelo de disco, diretamente da Habilitationschrift de Riemann, teve um impacto muito menor. Em certo sentido, o primeiro artigo, Saggio di Interprazione della Geometria non-euclidea, foi escrito sob a influência de Gauss, e o segundo, Teoria fondamentale degli spazi di curvatura costante, sob a influência de Riemann. (ARCOZZI, 2012, p. 7, tradução nossa)

No Saggio, Beltrami considerou a métrica

$$
\mathrm{ds}^{2}=\mathrm{R}^{2} \frac{\left(\mathrm{a}^{2}-\mathrm{v}^{2}\right) \mathrm{du^{2 }}+2 \mathrm{uvdudv}+\left(\mathrm{a}^{2}-\mathrm{u}^{2}\right) d v^{2}}{\left(\mathrm{a}^{2}-\mathrm{u}^{2}-\mathrm{v}^{2}\right)^{2}},
$$

com a observação de que é possível obtê-la da métrica (1), ao considerarmos o raio da esfera sendo um número imaginário iR e a constante ia em substituição a $\mathrm{R}$ e a, respectivamente, o que corrobora o ponto de vista de Lambert (vide [21], p. 375-376) e Taurinus (vide [8], p. 82), com a afirmação de que a geometria não euclidiana é a geometria de uma esfera de raio imaginário.

Em seguida, ele afirmou que a curvatura de uma superfície que admitisse a métrica (2) é -1/R ${ }^{2}$, o que poderia ser verificado diretamente da métrica. Tais superfícies de curvatura constante e negativa foram chamadas por ele de pseudoesferas.

As equações para as geodésicas de (2) são idênticas às geradas pela equação (1), de modo que suas geodésicas são linhas retas. Além disso, a equação (2) só define uma métrica no disco $\mathrm{u}^{2}+\mathrm{v}^{2}<\mathrm{a}$. Desse modo, a geometria em questão é aquela das cordas do disco de raio a, que viria a ser conhecida posteriormente por modelo do disco de Klein.

Os resultados no Saggio indicam que Beltrami buscava esclarecer duas (vide [2], p.10) situações:

1. Mostrar que o seu modelo correspondia de fato à geometria axiomática não euclidiana desenvolvida por Bolyai, Lobachevsky e seus predecessores.

Beltrami resolveu esse ponto mostrando diversas propriedades fundamentais da geometria, como a existência de uma única geodésica por dois pontos e propriedades referentes às isometrias do modelo, além de propriedades fundamentais da geometria não euclidiana, como aquelas referentes à soma dos ângulos de triângulos geodésicos, ângulo de paralelismo e horociclos.

2. Mostrar que o seu modelo correspondia a alguma superfície do mundo real.

Reescrevendo sua métrica através de mudanças de coordenadas e isometrias, Beltrami pôde entendêla como uma métrica que se obtém da rotação de curvas no espaço euclidiano. Ele fez isso de três maneiras distintas, e a primeira das superfícies obtidas, a tractroide, é chamada atualmente de pseudoesfera.

As superfícies de Beltrami possuíam uma inconveniência, suas geodésicas não poderiam ser prolongadas infinitamente, pois apresentavam arestas. Entretanto, na verdade, essa falha era inevitável. Após 33 anos, o matemático alemão David Hilbert (1862 - 1943) viria a provar que é impossível uma superfície completa (imersa) em R3 de curvatura constante e negativa. 
Figura 3: A tractroide, superfície gerada pela rotação da tractriz, conhecida atualmente por pseudoesfera.

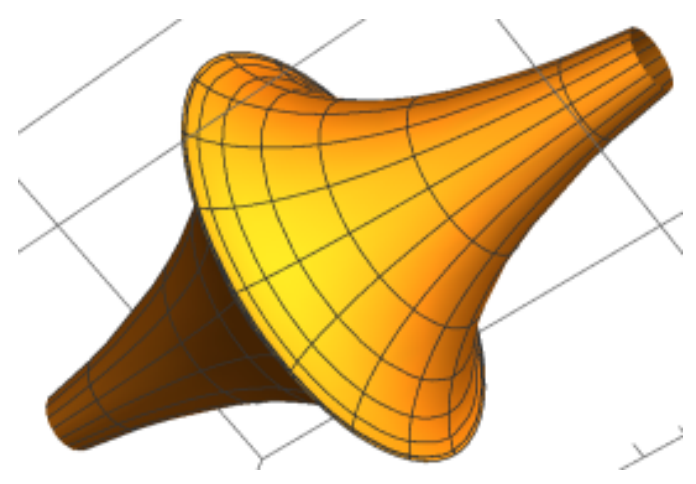

Assim, Beltrami materializou a geometria não euclidiana na geometria de uma superfície real, na qual valem os teoremas e resultados contraintuitivos que desconcertaram matemáticos por tanto tempo.

Ele provou, colateralmente, que o quinto postulado é independente dos demais, pois existe uma geometria na qual se verifica a sua validade. Sobre esse ponto, porém, não acreditamos que Beltrami considerasse ter demonstrado a consistência axiomática da geometria não euclidiana no sentido moderno ([21], p. 432). Todavia, muitos autores posteriores reconheceram que ele havia provado sua consistência, pois o modelo de Beltrami, com suas geodésicas dadas por cordas no disco de raio a, é compatível com os demais axiomas e qualquer problema nessa geometria pode ser traduzido em um outro problema da geometria euclidiana, ou seja, a geometria não euclidiana era tão consistente quanto a geometria euclidiana.

O Saggio teve como base a teoria de Gauss para superfícies (de dimensão 2). Todavia, em 1869, ele escreveu um segundo artigo sobre as geometrias não euclidianas, chamado de Teoria fondamentale degli spazii di curvatura costante, o qual chamaremos apenas de Teoria. Nesse trabalho ele considerou superfícies de dimensão arbitrária n, influenciado pela legendária aula inaugural de Riemann de 1854, mas somente publicada em $1867^{4}$ ([20]), onde Riemann apresentou ao mundo uma teoria que permitia descrever superfícies de qualquer dimensão.

O ponto de virada veio em 1868, com a publicação de dois trabalhos de E. Beltrami. No primeiro, Beltrami mostrou que a geometria não euclidiana bidimensional é nada mais nada menos do que o estudo de superfícies de curvatura negativa e constante. Introduziu o termo pseudoesfera de raio $R$ para uma superfície de curvatura $-1 / \mathrm{R}^{2}$. Na prática, ele usou o termo "pseudoesfera" apenas para superfícies completas, simplesmente conexas. Nesse primeiro artigo, ele ficou perplexo com o caso tridimensional. No entanto, depois de encontrar o discurso inaugural de Riemann, ministrado em 1854, mas publicado apenas após sua morte, em 1868, Beltrami publicou um segundo artigo, sobre geometria pseudoesférica n-dimensional. (MILNOR, 1982, p. 11, tradução nossa)

No Teoria, Beltrami considerou o hemisfério $\mathrm{x}^{2}+\mathrm{x}_{1}^{2}+\mathrm{x}_{2}^{2}+\cdots+\mathrm{x}_{\mathrm{n}}^{2}=\mathrm{a}^{2}$, com $\mathrm{x}>0$ no espaço $\mathrm{n}+1$

\footnotetext{
${ }^{4} \mathrm{O}$ ano impresso no artigo é 1867 , mas algums fontes indicam que ele só foi publicado de fato em 1868.
} 
dimensional, provido com a métrica

$$
d s=R \frac{\sqrt{d x^{2}+d x_{1}^{2}+d x_{2}^{2}+\cdots+d x_{n}^{2}}}{x} .
$$

$O$ hemisfério pode ser parametrizado pela função $x=\sqrt{a^{2}-x_{1}^{2}-x_{2}^{2}-\cdots-x_{n}^{2}}$, definida no disco $\mathrm{x}_{1}^{2}+\mathrm{x}_{2}^{2}+\cdots \mathrm{x}_{\mathrm{n}}^{2} \leq \mathrm{a}^{2}$, e Beltrami mostrou que as geodésicas desse mapa são os segmentos de reta do disco. Além disso, ele concluiu que a fronteira $x_{1}^{2}+x_{2}^{2}+\cdots x_{n}^{2}=a^{2}, x=0$, está a uma distância infinita dos pontos do interior do disco e discorreu sobre diversas propriedades das geodésicas e suas relações com os pontos no infinito, de acordo com a nomenclatura de Milnor [15] para os pontos na fronteira desse disco. Desse modo, Beltrami obteve o modelo que viria a ser conhecido como disco de Klein também para superfícies n dimensionais. Assim como Arcozzi ([2], p. 3), acreditamos que a nomenclatura mais adequada para o modelo seria modelo do disco (projetivo) de Beltrami-Klein.

Figura 4: A projeção ortogonal do hemisfério no plano (fonte: STILLWELL, 2010, p.370)

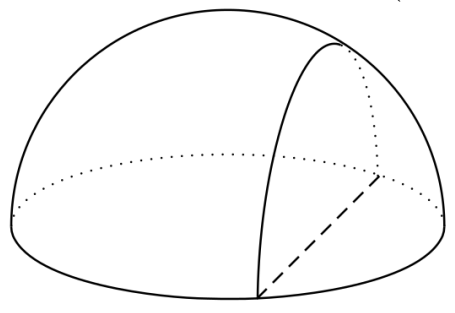

Fazendo algumas mudanças de variáveis, Beltrami reescreveu sua métrica como:

$$
\mathrm{ds}=\mathrm{R} \frac{\sqrt{\mathrm{d} \zeta_{1}^{2}+\mathrm{d} \zeta_{2}^{2}+\cdots+\mathrm{d} \zeta_{\mathrm{n}}^{2}}}{1-\frac{1}{4 \mathrm{R}^{2}}\left(\zeta_{1}^{2}+\zeta_{2}^{2}+\cdots+\zeta_{\mathrm{n}}^{2}\right)},
$$

a qual, segundo ele, poderia ser chamada de esterográfica, e comenta que essa métrica é análoga à apresentada no trabalho póstumo da aula inaugural de Riemann [20]. Tal métrica poderia ser obtida pela projeção estereográfica do hemisfério superior com $\mathrm{a}=\mathrm{R}$ no plano $\mathrm{x}=\mathrm{R}$ a partir do polo sul.

Figura 5: A projeção estereográfica do hemisfério em um plano paralelo ao seu equador (fonte: STILLWELL, 2010, p.370)

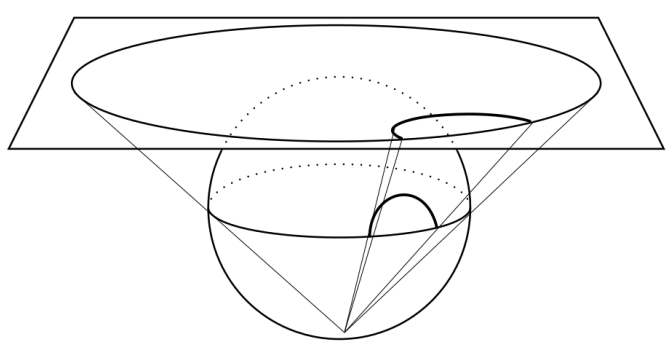


Beltrami mostrou que o hemisfério teria então a curvatura constante $1 / \mathrm{R}^{2}$, mas não apresentou uma exploração das geodésicas no sentido da geometria axiomática de seus predecessores. $\mathrm{O}$ disco no qual o hemisfério é projetado com a métrica acima, viria a ser conhecido por modelo do disco de Poincaré. Novamente, assim como Arcozzi ([2], p.3), acreditamos que a nomenclatura mais adequada seria modelo do disco (conformal) de Riemann-Beltrami-Poincaré.

Beltrami faz ainda outra mudança de variáveis, que corresponde a uma inversão na fronteira do disco e obtém a métrica:

$$
\mathrm{ds}=\mathrm{R} \frac{\sqrt{\mathrm{d} \eta^{2}+\mathrm{d} \eta_{1}^{2}+\mathrm{d} \eta_{2}^{2}+\cdots+\mathrm{d} \eta_{\mathrm{n}-1}^{2}}}{\eta} .
$$

Tal métrica corresponde àquela obtida pela projeção estereográfica do hemisfério superior com $\mathrm{a}=\mathrm{R}$ no semiplano $\mathrm{x}_{\mathrm{n}}=-\mathrm{R}, \mathrm{x}>0$, a partir do ponto $(0,0, \ldots, 0, \mathrm{R})$. Esse modelo viria a ser conhecido por modelo do semiplano de Poincaré. Mais uma vez, assim como Arcozzi ([2], p. 3 ), acreditamos que a nomenclatura mais adequada seria modelo do semiplano (conformal) de Liouville-Beltrami.

Figura 6: A projeção estereográfica do hemisfério em um plano perpendicular ao seu equador (fonte: STILLWELL, 2010, p.371)

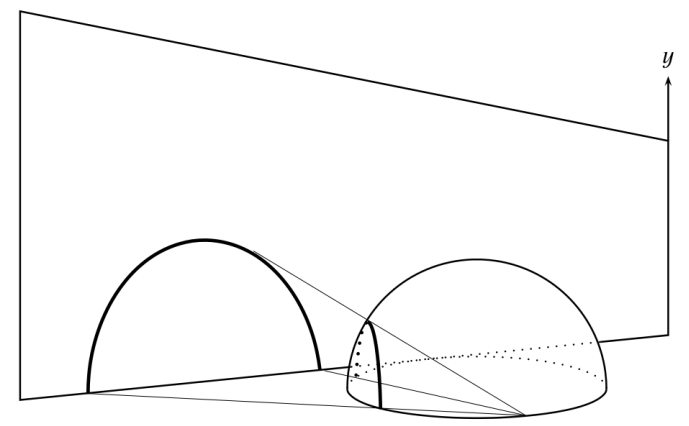

Após apresentar seus modelos, Beltrami discutiu algumas propriedades das geodésicas, como o fato de ser possível ligar quaisquer dois pontos nos modelos por uma geodésica (os modelos são simplesmente conexos). Aqui, destacamos que a ausência de discussões sobre polígonos e outras construções planas talvez já indicasse a mudança, no paradigma da geometria, de figuras construídas por retas e planos para curvas e superfícies diferenciáveis.

Assim, o mundo da geometria não euclidiana foi materializado na geometria de superfícies concretas, como destacado em [2]:

(4) Mais importante e duradouro, o universo da geometria não euclidiana não era mais o mundo contraintuitivo descrito por Lobachevsky e Bolyai: qualquer pessoa instruída sobre a teoria gaussiana das superfícies poderia calcular todas as consequências dos princípios não euclidianos diretamente a partir dos modelos de Beltrami; esse legado é bastante evidente até os dias atuais. (ARCOZZI, 2012, p. 2, tradução nossa)

Sobre a interpretação dos resultados, ressaltamos alguns fatos. O modelo do disco de BeltramiKlein foi obtido pela projeção ortogonal do hemisfério no plano de seu equador (base) e as geodésicas 
são segmentos do disco na projeção. Dessa maneira, as imagens das geodésicas no hemisfério são as curvas na interseção do hemisfério com planos perpendiculares à base e, portanto, são os arcos de circunferência perpendiculares à borda do hemisfério. Já os horociclos são as circunferências do hemisfério que passam por um ponto do seu equador.

Como as projeções estereográficas levam retas e círculos em retas ou círculos, as geodésicas nos modelos conformais do disco de Riemann-Beltrami-Poincaré e do semiplano de Liouville-Beltrami são dadas por segmentos ou semirretas perpendiculares às respectivas fronteiras ou por arcos de circunferência perpendiculares às fronteiras.

Nos modelos dos discos de Beltrami-Klein e de Riemann-Beltrami-Poincaré, os horociclos são circunferências que tangenciam a fronteira; já no modelo do semiplano de Liouville-Beltrami, os horociclos são as circunferências que tangenciam a reta $\mathrm{x}=0$ (ou o hiperplano, para dimensão maior do que 3) ou retas paralelas à reta $\mathrm{x}=0$ (ou ao hiperplano, para dimensão maior do que 3).

Beltrami fez duas observações para fechar o Teoria. A primeira delas diz que a geometria interna dos horociclos corresponde à geometria euclidiana, pois as retas paralelas à reta $\mathrm{x}=0$ (ou hiperplanos, se a dimensão é maior do que dois) têm métrica proporcional à euclidiana no modelo do semiplano. Na segunda observação, ele mostra que as circunferências geodésicas (ou hiperesferas), se a dimensão é maior do que dois) têm curvatura constante e positiva, além do fato de a geometria interna de suas geodésicas corresponder à geometria esférica.

\section{A geometria hiperbólica pode reclamar o título de geometria "universal", pois ela con- tém as outras duas geometrias de curvatura constante de modo natural. (STILLWELL, 1996, tradução nossa, p. 38)}

\section{Klein e Poincaré}

Na renascença italiana surgiu o método do desenho em perspectiva, fundamental para a representação adequada de figuras arquitetônicas e de objetos a partir de diferentes pontos de vista. Tais representações apresentam um efeito colateral: retas paralelas encontram-se em pontos no infinito e esses pontos determinam os horizontes das representações. O estudo da geometria dessas projeções tornou-se relevante e os matemáticos franceses Girard Desargues (1591-1661) e Blaise Pascal (1623-1662) realizaram vários avanços no estudo das propriedades invariantes por mapeamentos em perspectiva.

O matemático inglês Arthur Cayley (1821 - 1895) introduziu uma forma de calcular distâncias nessas projeções, permitindo estudar os espaços de projeção. Em seu método, fixa-se uma quádrica, chamada de absoluta, que representa os pontos no infinito e consideram-se as distâncias entre os pontos a e b no interior dessa quádrica como o logaritmo da razão harmônica:

$$
\mathrm{d}(\mathrm{a}, \mathrm{b})=\mathrm{C} \log \frac{|\mathrm{Aq}||\mathrm{Bp}|}{|\mathrm{Ap}||\mathrm{Bq}|} .
$$

O matemático alemão Felix Klein $(1849$ - 1925) considerou a constante $\mathrm{C}=1 / 2$ na geometria de Cayley e mostrou que essa é a geometria do modelo obtido por Beltrami no Saggio. Klein também introduziu o termo geometria hiperbólica ([13], p. 577) em referência ao fato de os pontos no infinito das retas serem reais ${ }^{5}$.

\footnotetext{
${ }^{5}$ Em referência ao uso dos termos elíptico e hiperbólico para involuções pelo matemático suiço Jakob Steiner 1796 $-1863$
} 
Figura 7: Uma circunferência como absoluta, e os pontos p e q na interseção da reta por A e B com a absoluta.

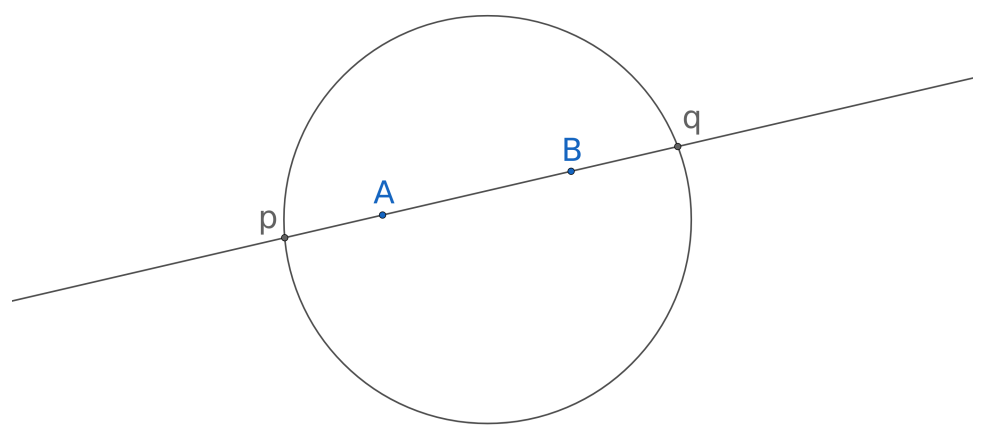

Poincaré reintroduziu os modelos apresentados no Teoria em [18] ao estudar o que seriam os movimentos rígidos do plano hiperbólico, ou seja, as isometrias que preservam áreas, ângulos e a orientação. Ele pôde representá-las por quocientes de funções complexas de determinante $\mathrm{ad}-\mathrm{bc}=1$, no modelo do hiperplano, através da função

$$
\mathrm{z} \rightarrow \frac{\mathrm{az}+\mathrm{b}}{\mathrm{cz}+\mathrm{d}}
$$

e no modelo do disco conformal, através da função complexa

$$
\mathrm{z} \rightarrow \frac{\mathrm{az}+\mathrm{b}}{\mathrm{c} \overline{\mathrm{z}}+\mathrm{d}}
$$

O modelo do Saggio é chamado, geralmente, de modelo de Klein, e os dois modelos do Teoria são frequentemente creditados a Poincaré. Há razões para isso. Klein tornou mais explícitas as conexões entre o modelo do Saggio e a geometria projetiva, a qual Beltrami só havia mencionado em seu artigo. Poincaré, como eu disse acima, foi o primeiro a usar os outros dois modelos para entender fenômenos aparentemente distantes do tópico não euclidiano. (ARCOZZI, 2012, tradução nossa, p. 3)

Por fim, ainda retornaremos ao papel da geometria esférica, que tem aplicações importantes na astronomia e na navegação, mas que ficou de lado na discussão do quinto postulado por apresentar múltiplas contradições e inadequações aos postulados de Euclides. Klein ainda conseguiu (em [13]) revisitá-la através da geometria elíptica, que pode ser vista como a geometria de uma esfera com pontos antípodas identificados.

\section{A visão moderna do espaço hiperbólico: o modelo do hiperboloide.}

Reynolds argumenta ([19], p. 442) que dificilmente alguém sustentaria que é melhor aprender geometria esférica a partir de um mapa do que a partir do globo, mas que quase todas as introduções à geometria hiperbólica apresentam modelos planos. É possível, e talvez seja a maneira mais 
natural, visualizar a geometria hiperbólica como a geometria da superfície de um hiperboloide. Porém, para isso é necessário trocar o produto interno euclidiano por outro.

O termo geometria hiperbólica foi introduzido por Klein em 1871, mas sem explicitar o hiperboloide como modelo. O matemático alemão Karl Weierstrass (1815 - 1897) e Poincaré fizeram menção às coordenadas do tipo $-\mathrm{x}^{2}+\mathrm{y}^{2}+\mathrm{z}^{2}=-\mathrm{R}^{2}$, mas uma referência explícita ao hiperboloide veio somente com o matemático alemão Wilhelm Killing (1847 - 1923), em 1885. Mergulhado no espaço

Figura 8: Folha superior do hiperboloide $H^{2}=\left\{(x, y, z) \in \mathbb{R}^{3} \mid-x^{2}+y^{2}+z^{2}=-R^{2}\right\}$

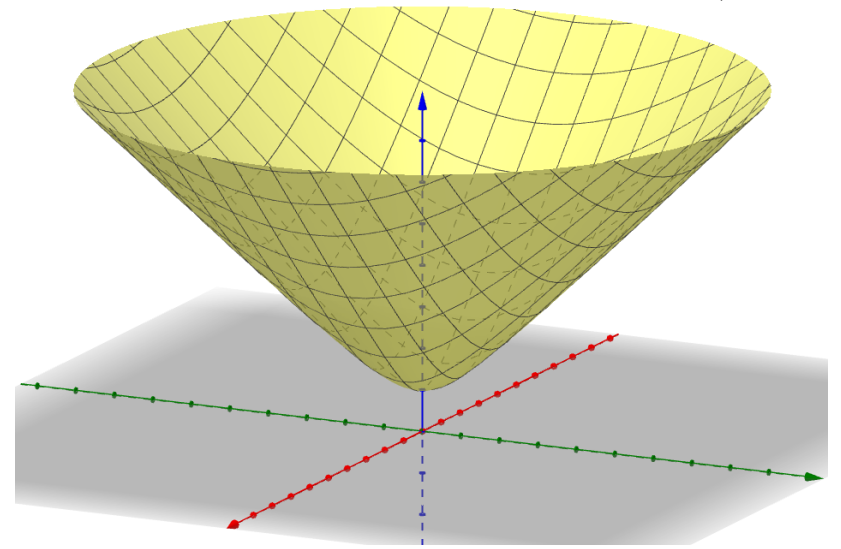

euclidiano, o hiperboloide não tem a métrica da geometria hiperbólica. Todavia, ao considerarmos o produto interno lorenziano:

$$
\left\langle\left(\mathrm{x}_{1}, \mathrm{y}_{1}, \mathrm{z}_{1}\right),\left(\mathrm{x}_{2}, \mathrm{y}_{2}, \mathrm{z}_{2}\right)\right\rangle=-\mathrm{x}_{1} \mathrm{x}_{2}+\mathrm{y}_{1} \mathrm{y}_{2},+\mathrm{z}_{1} \mathrm{z}_{2}
$$

a métrica induzida no hiperboloide torna-o um modelo da geometria hiperbólica.

A equação (7) não define um produto interno no sentido usual, mas um produto interno lorenziano, todavia a métrica induzida por ele é uma métrica no sentido usual e torna o hiperboloide uma superfície natural para a geometria hiperbólica, assim como a esfera é a superfície mais natural para a geometria elíptica. O uso desse produto interno veio naturalmente após a introdução da relatividade especial no início do século XX, compreendendo as três dimensões espaciais com "sinal" positivo e a quarta dimensão, o tempo, com "sinal" negativo.

Chamamos espaço de Minkowski de dimensão 3 a $\mathbb{R}^{3}$ munido com o produto interno da Equação (7). O espaço euclidiano é o nome dado para $\mathbb{R}^{3}$ munido do produto interno usual. O papel do hiperboloide (de dimensão n) no espaço de Minkowski é similar ao das esferas no espaço euclidiano.

Assim como a esfera apresenta um grupo considerável de simetrias no espaço euclidiano, o hiperboloide também possui muitas simetrias naturais. A saber, temos três tipos de simetrias. As reflexões, que preservam o eixo vertical e induzem bijeções do hiperboloide nele mesmo, assim como as rotações de um ângulo $\theta$ em torno do eixo vertical. O terceiro tipo de simetria é formado pelas transformações de Lorentz, que fazem o hiperboloide "deslizar" sobre ele mesmo. Essas simetrias estão representadas na Figura 9, sendo possível mostrar que as simetrias do hiperboloide são geradas por composições desses três tipos de simetrias. 
Figura 9: Ilustrações das simetrias do hiperboloide (reflexão por um plano que contém o eixo, rotação em um plano perpendicular ao eixo e uma transformação de Lorentz).
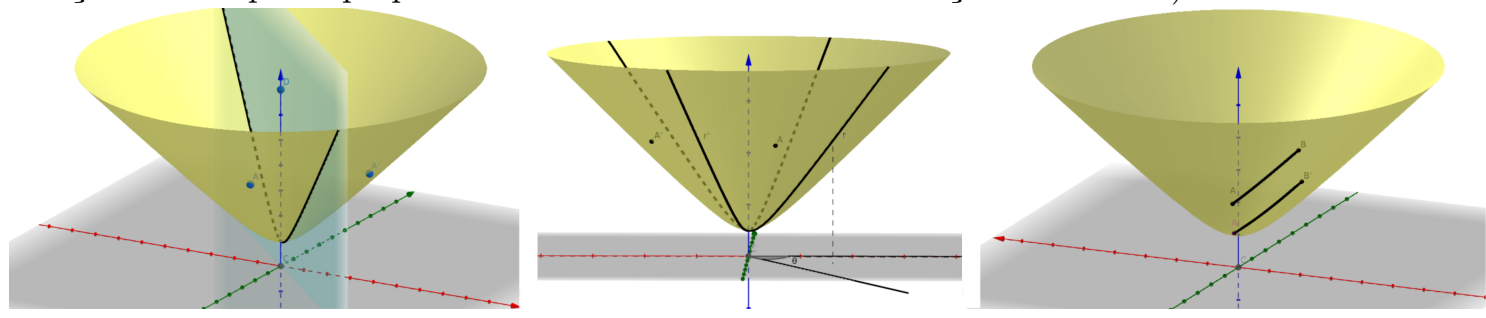

Considerando as simetrias do hiperboloide e a unicidade (local) da geodésica por dois pontos, é possível mostrar que elas estão na interseção do hiperboloide com planos que passam pela origem.

Figura 10: Uma geodésica obtida na interseção do hiperboloide com um plano pela origem.

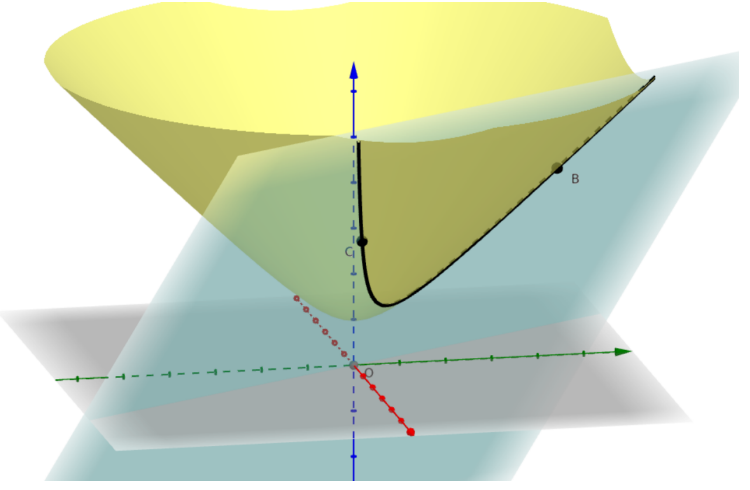

O modelo de Beltrami-Klein pode ser obtido naturalmente do hiperboloide ao projetarmos $\mathbb{H}^{2}$ no plano $\mathrm{x}=\mathrm{a}$, com a $>0$, usando a origem de $\mathbb{R}^{3}$ como centro de projeção.

Figura 11: O modelo obtido ao realizarmos a projeção do hiperboloide no plano que passa pelo seu polo a partir da origem.

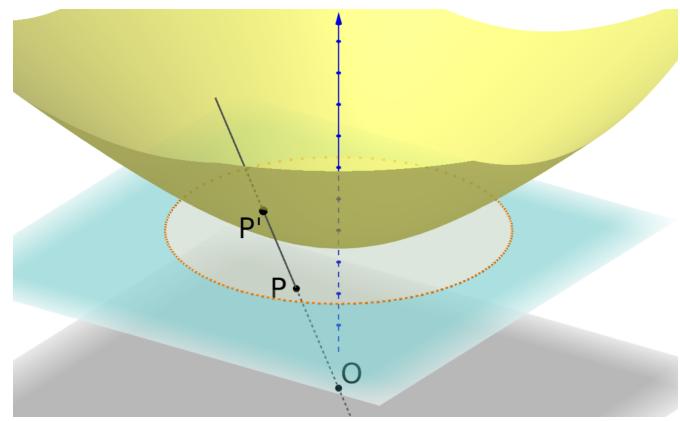

A projeção com centro $\mathrm{O}$ no plano $\mathrm{x}=\mathrm{a}>0$ leva qualquer plano que passa pela origem na sua interseção com o plano de projeção. Agora, levando em conta que as geodésicas do hiperboloide $\mathbb{H}^{2}$ são obtidas pela interseção do hiperboloide com planos que passam pela origem, vemos que 
elas serão projetadas sobre retas no plano de projeção (de modo análogo ao que acontece para as projeções centrais da esfera). Assim, as imagens das geodésicas por essa projeção são segmentos de reta.

Já a métrica (para $\mathrm{R}=1$ ), que define o modelo do disco de Riemann-Beltrami-Poincaré, pode ser obtida do hiperboloide ao projetarmos $\mathbb{H}^{2}$ no plano $\mathrm{x}=0$, usando como centro de projeção o "polo sul", $\mathrm{S}=(-1,0,0)$ de $\mathbb{H}^{2}$.

Figura 12: O modelo obtido ao realizarmos a projeção do hiperboloide no plano que passa pela origem a partir do polo sul.

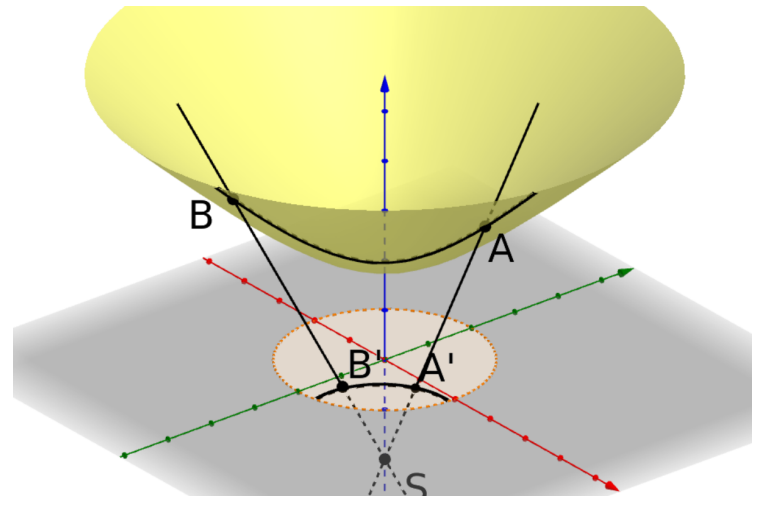

É possível calcular diretamente que suas geodésicas são projetadas sobre arcos de circunferência ortogonais à fronteira do disco.

Já o modelo do semiplano (conformal) de Liouville-Beltrami pode ser obtido ao invertermos o disco de Riemann-Beltrami-Poincaré por uma circunferência com o dobro do raio e com um ponto de tangência em comum.

Figura 13: A representação do disco de Riemann-Beltrami-Poincaré na circunferência por O, B e $\mathrm{D}$ é invertida pela circunferência maior gerando o semiplano da direita.

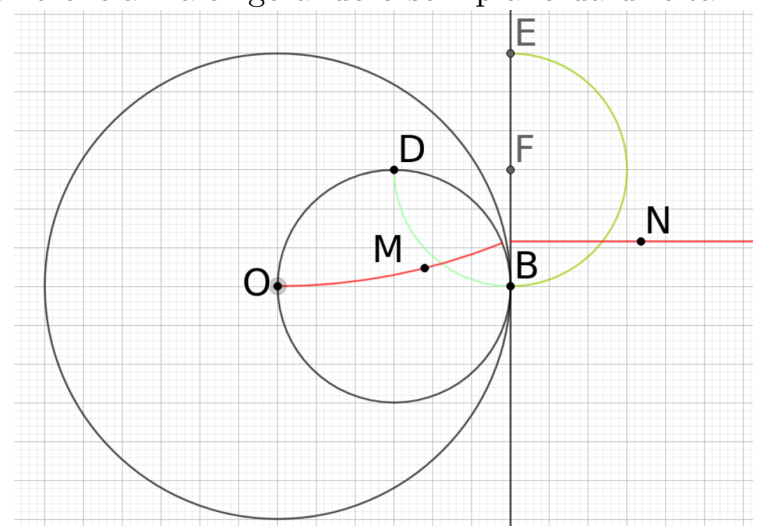

Como inversões levam circunferências em circunferências (degeneradas ou não), temos que as geodésicas do modelo do semiplano de Beltrami-Poincaré são arcos de circunferência ou semirretas 
ortogonais à reta que define o semiplano.

\section{Conclusão}

O desenvolvimento da geometria diferencial foi fundamental para o reconhecimento e fundamentação das geometrias não euclidianas, tirando-as do formalismo axiomático e tornando-as concretas. Essa materialização foi realizada na primeira vez por Eugenio Beltrami, quando investigava superfícies de curvatura constante. Apesar disso, seu nome acabou ficando em segundo plano na história da formação dessas geometrias. Também é frequente a omissão na conexão da geometrias não euclidianas com a geometria diferencial.

O trabalho empenha-se em divulgar as contribuições de Beltrami na longa história das geometrias não euclidianas, pois na maioria dos materiais produzidos e publicados em língua portuguesa sobre elas sentimos falta de menções ao seu nome, como, também, dos princípios da geometria diferencial que possibilitaram o desenvolvimento dessa nova geometria. Julgamos que um dos motivos para isso seja a simplicidade da definição de distância possível através da interpretação do disco de Beltrami-Klein com a geometria de Cayley, pela qual Klein tem todo o mérito, em oposição à formulação geométrica de Gauss e Riemann.

Muitas vezes as explorações das geometrias não euclidianas nos ensinos fundamental e médio reduzse a um exemplo de uma geometria diferente da euclidiana, pura e simplesmente como sua negação, sem motivo de ser. O trabalho busca elucidar algumas etapas do desenvolvimento das geometrias não euclidianas e contextualizá-las no restante da Matemática.

Por fim, a formulação moderna permite enxergar, de modo relativamente simples, os três modelos mais famosos de geometria hiperbólica como projeções de uma superfície concreta, o hiperboloide, que tem sua importância em aplicações da relatividade geral.

\section{Referências}

[1] Alves, S., Filho, L. C. S. "Encontro com o Mundo Não euclidiano"RPM 78, 2012.

[2] Arcozzi, N. "Beltrami's models of non-Euclidean geometry." In: Mathematicians in Bologna 1861-1960. p. 1-30. Basel: Birkhäuser, 2012.

[3] Artmann, B. "Projective geometry". In: Encyclopæedia Britannica. Disponível em: https:// www.britannica.com/science/projective-geometry. Acesso em 06 de mar. 2019.

[4] Beltrami, E. "Risoluzione del problema: "Riportare i punti di una superficie sopra un piano in modo che le linee geodetiche vengano rappresentate da linee rette". In: Annali di Matematica pura ed applicata, vol. I, n⿳⺈ 7, p. 185-204, 1865.

[5] Beltrami, E. 1868. "Saggio di interpretazione della geometria non-euclidea." In: Giornale di Matematiche, vol. VI, p. 284-312, 1868

[6] Beltrami, E. Teoria fondamentale degli spazii di curvatura costante. In: Giornale di Matematiche, vol. II, n⿳⺈ II, p. 232-255. 1869.

[7] Bicudo, I. Elementos/Euclides. São Paulo: Unesp, 2009.

[8] Bonola, R. Non-Euclidean Geometry. Chicago, Open Court Publishing Company, 1912.

[9] Carmo, M. P. “Geometrias não euclidianas." In: Matemática Universitária, no 6, p. 25-48, 1987.

[10] Carrera, J. P. Euclides: A geometria. Lisboa: National Geographic, 2012. 
[11] Coxeter, H. S. M.Non-Euclidean Geometry. Washington: The Mathematical Association of America, 6 ${ }^{\mathbf{a}}$ edição, 1998.

[12] Garbi, G. G. A Rainha das Ciências: um passeio histórico pelo maravilhoso mundo da matemática. São Paulo: Livraria da Física, 2006.

[13] Klein, F. Ueber die sogenannte Nicht-Euklidische Geometrie. Mathematische Annalen, 1871. Disponível em: http://eudml.org/doc/156553. Acesso em 20 de fev. 2019.

[14] Lagrange, J. L. 1779. "Sur la construction des cartes géographiques." In: Mémoires de l'Académie royale des Sciences et Belles-Lettres de Berlin, Berlin, 1779.

[15] Milnor, J. 1982. "Hyperbolic Geometry: the first 150 years." In: Bulletin Of The American Mathematical Society, no 1, p. 9-24, 1982.

[16] Mlodinow, L. A janela de Euclides. São Paulo: Geração Editorial, 2008.

[17] Oliva, W. M. "A independência do axioma das paralelas e as Geometrias não euclidianas." $R P M$ 2, p. 28-31, 1983.

[18] Poincaré, H. Théorie des groupes fuchsiens. 1882. Disponível em: https://projecteuclid.org/ euclid.acta/1485801055. Acesso em 10 de abr. 2019.

[19] Reynolds, W. F. "Hyperbolic Geometry on a Hyperboloid." The American Mathematical Monthly, vol. 100, No. 5, pp. 442-455, 1993.

[20] Riemann, B. (publicada por Richard Dedekind após a morte de Riemann). "Ueber die Hypothesen, welche die Geometrie zu Grunde liegen." Abhandlungen der Königlichen Gesellschaft der Wissenschaften zu Göttingen, vol. 13, 1867.

[21] Scriba, C. J. e Schreiber, P. 5000 years of geometry: mathematics in history and culture. Basel: Springer-Verlag, 2015.

Márcio Rostirolla Adames

Universidade Tecnológica Federal do Paraná <marcioadames@utfpr.edu.br>

Fernanda Mocelin Schena Colégio Nossa Senhora de Sion $<$ ferschena@yahoo.com.br>

Recebido: 15/10/2020

Publicado: 20/09/2021 